\title{
THE DECOMPRESSION OF COLD NEUTRON STAR MATTER
}

\author{
JAMES M. LATTIMER \\ The University of Texas; and The Enrico Fermi Institute, University of Chicago \\ Fred Mackie and D. G. Ravenhall \\ The University of Illinois \\ AND \\ D. N. SCHRAMM \\ The Enrico Fermi Institute, University of Chicago \\ Received 1976 August 16
}

\begin{abstract}
The composition of expanding, initially cold, neutron star matter is examined. A semiempirical mass formula for nuclear matter is developed. Under the assumption that the matter occupies its lowest energy state, the four equilibrium conditions which determine the composition of the equilibrium, preexpansion matter are described. It is argued that as the density falls below nuclear density, nuclear clusters are formed which are very similar to those of equilibrium matter of the same density. The expansion is carried out by assuming that the matter inside the nucleus is in equilibrium with the dripped neutron sea outside. As the density is further lowered, these nuclei lose neutrons to the external sea of neutrons. Finally, when the $\beta$-decay time scale becomes shorter than the expansion time scale, $\beta$-decays take place. The nuclei then increase in both proton and neutron number, until they become unstable to fission. It is noted that these latter stages bear a resemblance to the classical $r$-process-and, in fact, as the matter cycles and the number of neutrons is depleted, the similarity increases. But because the path of the nuclei in the $Z-N$ plane lies somewhat more to the neutron-rich side of the valley of beta stability, it is easier to make superheavy elements with $Z \sim 126$ (which may have been recently detected) than in the $r$-process.
\end{abstract}

Subject headings: black holes - dense matter - nuclear reactions - stars: neutron

\section{INTRODUCTION}

Recently, some astrophysical processes have been investigated in which matter originally at nuclear densities or greater is suddenly expanded to much lower densities. For example, the tidal disruption of neutron stars by black holes (the only objects capable of generating large enough tidal forces) may occur in evolved neutron-starblack-hole binary systems. Lattimer and Schramm (1976) have found that close double stars containing at least one massive star may be able, in a large number of cases, to evolve into black-hole-neutron-star pairs via the processes of mass transfer and supernova. Furthermore, general considerations of mass transfer and mass loss during the evolution of these systems leads one to expect that the final configuration has a separation so small that gravitational radiation will cause a complete orbital decay, resulting in a black-hole-neutron-star collision. The tidal forces of the black holes are sufficiently strong to totally disrupt the neutron star before it falls within the event horizon of the black hole, leading to the ejection of some of the neutron star matter. Lattimer and Schramm (1976), considering the statistical frequency of these events, conclude that if all this ejecta were $r$-process matter, it would be possible to explain much of the $r$-process matter in the Galaxy by this mechanism. This results because their models indicate that up to $5 \%$ of the neutron star would be ejected.

Another scenario that has been proposed for expanding dense neutron star matter is a neutron star volcano (Dyson 1970). Changes in the internal structure of a neutron star as it cools or spins down produce stresses that perhaps are capable of eventually forcing some of the interior matter to the surface, or even beyond.

Other investigators have studied the ejection of hot neutron star matter following a supernova explosion (Cameron, Truran, and Delano 1970; Schramm 1973; Sato, Nalsayana, and Ikeuchi 1973; Sato 1974; Hillebrandt et al. 1976; Schramm and Norman 1976). Because the time scale for the neutrino cooling of a neutron star (Bahcall and Wolf 1965) is of the order of a million years or less, it is reasonable to assume that the matter in the neutron star before breakup is in its ground state (i.e., at zero temperature). It is the purpose of this paper to examine the ejection of cold neutron matter and to determine if the final composition of this matter may be similar to that normally associated with the hot, high-neutron-flux, $r$-process.

Cold neutron star matter can, for the purposes considered here, be divided into three regimes. The center, at densities greater than nuclear densities $\left(3-4 \times 10^{14} \mathrm{~g} \mathrm{~cm}^{-3}\right)$, is composed mostly of neutrons (or heavier baryons 
above $10^{15} \mathrm{~g} \mathrm{~cm}^{-3}$ ) with a few percent of protons, electrons, and muons (for a review of the structure of neutron stars, see Baym and Pethick 1975). Matter between neutron drip $\left(3 \times 10^{11} \mathrm{~g} \mathrm{~cm}^{-3}\right)$ and nuclear densities occupies the second region, and contains large nuclear clusters in a sea of degenerate neutrons and electrons. The outer crust of the neutron star, finally, comprises at most one thousandth of the total mass, and may therefore be neglected in this discussion.

For the ground-state matter that is initially more dense than nuclear densities, the fraction of protons does not significantly vary as a function of density. The matter ceases to be in beta equilibrium as the density is lowered, but the expansion time scale is too short for $\beta$-decays to occur. Thus when the density reaches $2 \times 10^{14} \mathrm{~g} \mathrm{~cm}^{-3}$, nuclear clusters are formed which should be very similar to the ground-state nuclei at this density.

The program for determining the final abundances is as follows: The composition of the equilibrium (prebreakup) matter is found by assuming the matter to be in its absolute ground state at a given density. This matter is then allowed to expand. The relevant physical processes such as $\beta$-decays and fissions are presumed to occur only if their time scales are less than the expansion time scale. The composition of the matter may then be found as a function of its density as it expands. The methods used are an extension of the equilibrium-matter calculations of Baym, Bethe, and Pethick (1971), as modified by Ravenhall, Bennett, and Pethick (1972). The sensitivity of $\beta$ decay and fission time scales to energy thresholds requires, however, that nuclear shell effects be added to the nuclear Hamiltonian. For the neutrons in the nucleus, the smearing out of the neutron shell-effects caused by the high density of external "dripped" neutrons is allowed for.

The process is found to evolve as follows. In the initial phase of the expansion, neutrons leave the nucleus rapidly, although the density of the dripped neutrons is also decreasing. Beta decay, which does not occur until the density has dropped to nearly $10^{11} \mathrm{~g} \mathrm{~cm}^{-3}$, reverses this trend. The temperature, which had remained zero (on a nuclear scale) during the initial expansion, now rises to about $10^{9} \mathrm{~K}$. When the proton number $Z$ of the nuclei approaches 120 , fission occurs, and the temperature increases dramatically. The high temperature, coupled with the relatively high dripped neutron density, supply the ingredients for a subsequent $r$-process phase of the kind described by Schramm (1973). Since our calculations are based on models of cold nuclear matter, however, this latter stage will not be explored in the present work. It is noted, though, that since the synthesis path found here lies to the neutronrich side of the $r$-process path, it is inherently easier to produce larger proton numbers than in the $r$-process because (1) the path terminates due to fission at a large $Z$, and (2) there must be a larger number of $\beta$-decays to bring the nuclei to beta stability. This is important since Gentry et al. (1976) have found evidence for superheavy nuclei with $Z \sim 126$ : previously it had been supposed that superheavies would have a proton number near 114 .

In § II, the semiempirical liquid drop model used for the nucleus is discussed. Section III details how the equilibrium composition of neutron star matter is determined using this model. The physical mechanisms taking place during the expansion are treated in $\S$ IV. The results of this model are given in $\S \mathrm{V}$. In $\S$ VI some concluding remarks and speculations are made.

\section{NUCLEAR MODEL}

A nuclear model is needed to describe the matter in the dripped neutron regime $\left(\rho=4 \times 10^{11}\right.$ to $2 \times 10^{14} \mathrm{~g}$ $\mathrm{cm}^{-3}$ ). In what follows, energies will be measured in $\mathrm{MeV}$, distances in $\mathrm{fm}$, and wavenumbers in fm ${ }^{-1}$. The average nucleus is characterized by its mass $A$, proton number $Z$, and radius $r$. The quantity $x=Z / A$ is a useful parameter which decreases monotonically with the density in this regime. The nuclear model assumed follows that of Baym, Bethe, and Pethick (1971), who consider the nucleus to be a compressible liquid drop, in a lattice and immersed in a sea of degenerate neutrons (at zero temperature), which is a reasonable approximation until the neutron gas heats up to $\sim 10^{9} \mathrm{~K}$. The total energy of a nucleus, including the lattice, is

$$
E_{\mathrm{nuc}}=\left[m_{n} c^{2}(1-x)+m_{p} c^{2} x+W\right] A+E_{\mathrm{Coul}}+E_{\mathrm{surf}}+S,
$$

where $m_{n}$ and $m_{p}$ are the masses, respectively, of the neutron and proton, $W$ is the bulk energy per nucleon of nuclear matter, $E_{\text {surf }}$ is the surface energy, and $E_{\text {coul }}$ is the total of all the Coulomb energy in the system (nuclear, lattice, and the proton-proton, proton-electron, and electron-electron exchange energies). $S$ is a semiempirical shell energy correction. These quantities depend on the nuclear parameters in a manner determined by the nuclear model.

The bulk energy of the nucleus is calculated, following Ravenhall, Bennett, and Pethick (1972), for a system of nucleons interacting through a contact pseudopotential (Skyrme interaction), with six parameters to fit ordinary nuclei. The particular Hamiltonian used follows closely the form given by Vautherin and Brink (1970) with slight modification of the isospin dependence in the three-body interaction to improve the agreement with the neutron gas results of Siemens and Pandharipande (1971), obtained using nuclear matter theory. The surface energy is obtained in the Hartree-Fock approximation using the same Hamiltonian (see Ravenhall, Bennett, and Pethick 1972). The nuclear Coulomb energy is just that of a uniformly charged sphere of total charge $Z e: \frac{3}{5} Z^{2} e^{2} / r$. The lattice energy is found in the Wigner-Seitz approximation (see, for example, Baym, Bethe, and Pethick 1971).

The semiempirical shell correction $S$ we choose is given by (Myers and Swiatecki 1966)

$$
\left.S(N, Z)=C[F(N)+F(Z)) /\left(\frac{1}{2} A\right)^{2 / 3}-c A^{1 / 3}\right]
$$


with $C=5.8 \mathrm{MeV}, c=0.26$, and

$$
F(N)=\frac{3}{5}\left[\left(M_{i}^{5 / 3}-M_{i-1}^{5 / 3}\right)\left(N-M_{i-1}\right) /\left(M_{i}-M_{i-1}\right)-\left(N^{5 / 3}-M_{i-1}{ }^{5 / 3}\right)\right] .
$$

The $M_{i}$ are the set of magic numbers, here taken to be

$$
M_{i}=\frac{1}{3}\left[(i+1)^{3}+5(i+1)\right]
$$

for $i \geq 3$, except for the proton magic number $M_{6}$ which is assumed to be 114. This formula works well for observed nuclei along the path of beta stability. Neutron star matter, however, contains nuclei located far from this path in general. Even the correct set of magic number is not known for the regions of interest. For example, for some densities 40 may be a magic proton number instead of 50 (Negele and Vautherin 1973). However, the important requirement is the qualitative effect shells have upon the expansion. Although the formula for $S(N, Z)$ may be wrong in its details, it does qualitatively provide the effects of shells.

Next, one must take into account the influence of the external degenerate neutron gas on the neutron energy levels. Consider a simple model of the broadening of those levels above the nuclear potential well due to the scattering of the neutron gas. Assuming the nucleus to be a square well, the width $\Gamma$ of a level above the nucleus is about $2 h^{2} k_{n} / m_{n} r$, where $k_{n}$ is the wavenumber of the external neutron gas. $\Gamma$ is about $10 \mathrm{MeV}$ near nuclear densities and about $1 \mathrm{MeV}$ near densities of $10^{11} \mathrm{~g} \mathrm{~cm}^{-3}$. Since the largest width between energy levels, which occurs at magic numbers, is about $1.5 \mathrm{MeV}$, it is clear that over much of the region of interest neutron shells do not occur as such, because of the smearing due to the outside neutron gas. However, near neutron drip densities the level structure will begin to be important. The above may be crudely approximated by replacing $F(N)$ and $c$ in equation (2) by

$$
\begin{gathered}
F^{\prime}(N)=F(N)\left[\exp \left(1-\Gamma / \Delta_{N}\right)-1\right] /(e-1), \\
c^{\prime}=c\left[\frac{F^{\prime}(N)}{F(N)}(1-x)+x\right]
\end{gathered}
$$

when $\Gamma \leq \Delta_{N} ; F^{\prime}(N)=0$ and $c^{\prime}=c x$ when $\Gamma>\Delta_{N} . \Delta_{N}$ is the neutron energy level spacing:

$$
\Delta_{N} \approx \partial^{2}[S(N, Z)+W A] / \partial N^{2} .
$$

Equation (5) has the effect of reducing the magnitude of the neutron shell energy when $\Gamma \neq 0$, while (6) keeps the average of $S(N, Z)$ near 0 .

The shell function $S(N, Z)$ has the appearance of a humped function with minima at magic numbers. The derivatives of $S$ at magic numbers are discontinuous; therefore, for computational purposes these minima have been smoothed out by requiring the first and second derivatives to be continuous.

When the shell function is near a maximum (i.e., between magic numbers) and exceeds a certain critical value, the spherical nucleus will be unstable, and will deform. The change in energy due to the deformation is treated as in Myers and Swiatecki (1966). The net effect of the deformation is to flatten out the humps in the shell function $S(N, Z)$.

A major step forward in the understanding of neutron star matter was the realization of Baym, Bethe, and Pethick (1971) that the energy of the dripped neutron gas, $W^{(d)}$, should be obtained from the same nuclear interaction which provides the energy of the matter in the nucleus. That feature is preserved here. The electron energy is computed assuming the electrons to be relativistic and degenerate.

\section{EQUILIBRIUM CONDITIONS}

It is necessary to calculate the equilibrium composition of the matter, the initial state of the expansion. As discussed by Baym, Bethe, and Pethick (1971), there are four equilibrium conditions that must obtain; they arise from the condition that the total energy per unit volume of the system must be a minimum at a given baryon density $n_{b}$. First, the number of nucleons in the nucleus must be optimized:

$$
\left.\frac{\partial\left(E_{\mathrm{nuc}} / A\right)}{\partial A}\right|_{x, n_{b}, r / R, k}=0
$$

is the required condition. Second, the nucleus must be in beta equilibrium, or

$$
\mu_{e}=\mu_{n}^{(N)}-\mu_{p}^{(N)}+\left(m_{n}-m_{p}\right) c^{2},
$$


where $\mu_{n}{ }^{(N)}$ and $\mu_{p}{ }^{(N)}$ are the chemical potentials of the neutrons and protons in the nucleus, not including the rest masses. These are evaluated in the form

$$
\begin{aligned}
& \mu_{n}^{(N)}=\left.\frac{\partial E_{\text {nuc }}}{\partial A}\right|_{z, r, k_{n}, R, E_{\text {surf }}}-m_{n} c^{2}, \\
& \mu_{p}^{(N)}=\left.\frac{\partial E_{\text {nuc }}}{\partial A}\right|_{N, r, k_{n}, R, E_{\text {surf }}}-m_{p} c^{2} .
\end{aligned}
$$

Next, the neutrons in the dripped region must be in equilibrium with those in the nucleus; that is, their chemical potentials must be the same. As a result, zero energy is involved when a neutron is transferred between the nucleus and the dripped region. One has

$$
\mu_{n}^{(N)}=\mu_{n}^{(d)}=\partial\left[W^{(d)} n_{d}\right] / \partial n_{d},
$$

where $n_{d}$ is the number density of dripped neutrons. Finally, the pressure of the external neutron gas, $P_{d}$, must be the same as that in the nucleus, $P_{N}$ :

$$
P_{N}=-\left.\frac{1}{4 \pi r^{2}} \frac{\partial E_{\mathrm{nuc}}}{\partial r}\right|_{z, A, n_{d}, R}=P_{d}=n_{d}^{2} \frac{\partial W^{(d)}}{\partial n_{d}} .
$$

\section{PHYSICAL PROCESSES IN THE DECOMPRESSION}

Once the equilibrium composition of the material as a function of density has been found, one may begin to expand the matter. The expansion of a unit cell of matter, containing one nucleus, is examined. The condition that the number of baryons in this cell remain constant will replace the first equilibrium condition, equation (8). Those other equilibrium conditions which are characterized by time scales short compared with the expansion time scale are assumed to remain satisfied during the decompression. The expansion time scale is on the order of a freefall time scale $\left(446 \rho^{-1 / 2} s\right)$. The two neutron phases will be in equilibrium as long as the neutron-capture time scale is shorter. Assume that the neutron capture cross section $\sigma$ of a nucleus times the average neutron velocity $V_{n}$ is $1 \mathrm{mb} \times 10^{7} \mathrm{~cm} \mathrm{~s}^{-1}$, a conservative estimate (Blake and Schramm 1973). The lifetime $\tau_{n}$ of a nucleus against capturing a neutron is

$$
\tau_{n}^{-1} \sim n_{d} \sigma V_{n} .
$$

The free neutron number density $n_{d}$ vanishes when $\rho \sim 10^{11} \mathrm{~g} \mathrm{~cm}^{-3}$. Assume that there remains only one free neutron per nucleus (average mass $A \sim 300$ ). Thus $n_{d}=10^{11} N_{\mathrm{A}} / 300 \sim 2 \times 10^{32} \mathrm{~cm}^{-3}$, where $N_{\mathrm{A}}$ is Avogadro's number. As a result, $\tau_{n}$ is always less than $5 \times 10^{-14} \mathrm{~s}$. This is about 10 orders of magnitude faster than the expansion time scale. A second equilibrating process, involving pressure readjustment, should take place on a time scale corresponding to the sound travel time across a nucleus. This time scale is much less than $\tau_{n}$. Thus the equilibrium conditions (12) and (13) are retained during the expansion.

Beta decays are allowed to occur only when two criteria are satisfied. The first is an energy threshold condition,

$$
\mu_{n}^{(N)}-\mu_{p}^{(N)}+\left(m_{n}-m_{p}\right) c^{2}-\mu_{e} \equiv \Delta>0 .
$$

In computing the shell energy contributions to this equation the unsmoothed Myers and Swiatecki mass formula is used. The second criterion is that the $\beta$-decay time scale must be less than the expansion time scale. The $\beta$-decay may be treated like that of an ordinary nucleus. Then the $\beta$-decay half-life is

$$
\tau_{\beta}{ }^{-1}=\frac{15 G^{2}}{\ln (2) \pi^{3}}\left(\left|M_{v}\right|^{2}+\epsilon^{2}\left|M_{A}\right|^{2}\right) \int_{0}^{E_{e \max }} \int_{E_{f e}}^{E} E^{\prime 2}\left(E-E^{\prime}\right)^{2} d E^{\prime} \rho(E) d E,
$$

where $G$ is the weak interaction coupling constant, $M_{v}$ and $M_{A}$ are the axial and polar vector components of the reduced nuclear matrix elements, $\epsilon \sim 1.21, \rho(E)$ is the density of states in the daughter nucleus, and $E_{\text {emax }}=\Delta+$ $\mu_{e}$. It has been assumed that $\hbar=c=1$ and that the electrons are relativistic and degenerate. This equation can be rewritten, after integrating, as

$$
\tau_{\beta}^{-1}=\langle\rho / f t\rangle(\ln 2)^{-1}\left(2.5 E_{f e}{ }^{2} \Delta^{4}+E_{f e} \Delta^{5}+\frac{1}{6} \Delta^{6}\right),
$$

where the density of states has been incorporated into the usual $f t$ value. Here, $f t=2 \pi^{3} G^{-2}\left(\left|M_{v}\right|^{2}+\alpha^{2}\left|M_{A}\right|^{2}\right)^{-1}$. In the absence of a detailed theoretical calculation for the matrix elements of extremely neutron-rich nuclei, $\log$ $\langle p / f t\rangle$ is taken to be in the range -2.8 to -5.5 .

It will be important to determine the approximate temperature that the expanding material attains, since the energies and pressures derived so far are valid only at zero temperature. The Debye temperature $\theta_{\mathrm{D}}$ is

$$
k_{\mathrm{B}} \theta_{\mathrm{D}}=\hbar Z e\left(A m_{p} R^{3} / 3\right)^{-1 / 2} \sim 13\left(A R^{3}\right)^{-1 / 2} Z \mathrm{MeV}
$$


(Lamb 1974), where $k_{\mathrm{B}}$ is the Boltzmann constant. Using $Z^{2} / A \sim 10$ and $R \sim 100 \mathrm{fm}$, one finds $\theta_{\mathrm{D}}$ to be $5 \times 10^{8} \mathrm{~K}$. Since the effective cutoff between low and high temperatures is $\theta=0.444 \theta_{D}$, and since the temperatures that we will be considering are of the order of $10^{9} \mathrm{~K}$, we take the high-temperature Debye approximation for the specific heat of the nuclei in the lattice, namely $3 k_{\mathrm{B}}$. For finite temperatures the additional thermal energy per nucleus, neglecting the energy density of radiation, is

$$
E_{T}=3 k_{\mathrm{B}} T+\chi_{n} k_{\mathrm{B}}^{2} T^{2} N_{d}+\chi_{e} k_{\mathrm{B}}^{2} T^{2} Z,
$$

where $N_{d}$ is the number of dripped neutrons per nucleus and $\chi_{n}$ and $\chi_{e}$ represent the lowest-order coefficients in a $T$ expansion for the partially degenerate neutron and electron Fermi gases. (We note that nuclear excited states should not contribute importantly to this relationship.) The neutrons are nonrelativistic, so $\chi_{n}=\left(\frac{1}{2}\right)^{5 / 3} m_{n} \pi^{2} /\left(\hbar k_{n}\right)^{2}$, while the electrons are relativistic, so $\chi_{e}=(18 Z)^{-1 / 3} \pi^{5 / 3} R / \hbar c$ (see Landau and Lifshitz 1969). It should be noted that $\chi_{n} N_{d} /\left(\chi_{e} Z\right) \sim 300$, so the electron contribution is small. For consistency in this expansion in temperature, if $\chi_{n}$ becomes greater than $\left(k_{\mathrm{B}} T\right)^{-1}, \chi_{n}$ is set equal to $\left(k_{\mathrm{B}} T\right)^{-1}$. With the introduction of a finite temperature, the additional pressure is given by $P_{T}=2 E_{T} / 3 V$, with $V$ the volume of the Wigner-Seitz cell.

To compute the temperature, one uses the first law:

$$
d\left(E_{\mathrm{nuc}}+W^{(d)} N_{d}+E_{e}\right)+d E_{T}+\left(P+P_{T}\right) d V=d Q,
$$

where $d Q$ represents the energy carried off by neutrinos. Neutrino cooling rates are too low to be of much importance. For instance, Bahcall and Wolf (1965) give for the modified Urca process

$$
-\frac{d Q_{\text {cool }}}{V d t}=6 \times 10^{14}\left(\rho / \rho_{\text {nuc }}\right)^{2 / 3} T_{9}^{8} \mathrm{MeV} \mathrm{fm}^{-3} \mathrm{~s}^{-1},
$$

where $T_{9}=T / 10^{9}$ and $\rho_{\text {nuc }}=3.7 \times 10^{14} \mathrm{~g} \mathrm{~cm}^{-3}$. Compared to the adiabatic cooling rate, $P d V / d t$, this value is generally small. Other cooling processes (electron bremsstrahlung [Dicus et al. 1976], plasmons [Beaudet, Petrosian, and Salpeter 1967], phonons [Flowers 1973]) give rates of the order of or less than equation (21) in the density regimes of interest. Neutrinos will also remove energy through $\beta$-decays. The average energy carried off by a neutrino is

$$
\begin{aligned}
-d Q_{\beta} & =\langle\rho / f t\rangle \tau_{\beta} \int_{0}^{E_{e \max }} \int_{E_{f e}}^{E_{e \max }-E}\left(E_{e \max }-E-E^{\prime}\right)^{3} E^{\prime 2} d E^{\prime} d E \\
& =\frac{3}{5} \Delta\left(1+\Delta / 3 \mu_{e}+\Delta^{2} / 21 \mu_{e}^{2}\right) /\left(1+2 \Delta / 5 \mu_{e}+\Delta^{2} / 15 \mu_{e}^{2}\right) .
\end{aligned}
$$

Combining the above equations, we obtain a differential equation for $T$.

It will be necessary to determine when a nucleus will fission; i.e., the fission time scale $\tau_{f}$ must be computed. In general, there are two processes of importance: spontaneous fission and neutron-induced fission. Following Schramm and Fowler (1971), the spontaneous fission half life may be estimated by the semiempirical formula

$$
\log _{10} \tau_{f .}=C_{1}\left(F+S(N, Z)\left(C_{2}+C_{3} F\right)+C_{6}\right) .
$$

This fission rate depends primarily on

$$
F=\left(Z^{2} / A\right)\left(1-\kappa I^{2} / A^{2}\right),
$$

where $I=N-Z$ and $\kappa$ is the ratio of the surface asymmetry coefficient to the surface term in the semiempirical mass formula. For the Hartree-Fock calculation of the surface energy by Ravenhall, Bennett, and Pethick (1972) that is used here, $\kappa=2.1$. One finds the constants $C_{i}$ by fitting the fission half-lives of known nuclei. For $\kappa=2.1$, one can estimate from Schramm and Fowler (1971) that $C_{1}=-4.32, C_{2}=-23.9, C_{3}=0.588$, and $C_{6}=-45.5$.

The rate of neutron-induced fission will be nearly instantaneous when the quantity $\theta=E_{n}-\mu_{n}-E_{b}$ is greater than zero, but decreases exponentially with decreasing $\theta$ (Schramm and Fowler 1971). $E_{n}$ is the thermal neutron energy; when $T=2 \times 10^{9} \mathrm{~K}, E_{n} \sim 0.2 \mathrm{MeV}$. The neutron binding energy $\mu_{n}$ is about $0.5 \mathrm{MeV}$ for the cases of interest. $E_{b}$ is the fission barrier; empirically (Myers and Swiatecki 1966) it is $\sim \frac{1}{8}\left(21.5+\log _{10} \tau_{f}\right) \geqslant 2.5 \mathrm{MeV}$ as long as $\tau_{f}>\tau_{n}$. Thus, since $\theta \approx-2.5 \mathrm{MeV}$ for the nuclei considered here, neutron-induced fission may not be important. This crude treatment of fission is certainly susceptible to quantitative error. However, it was found that the semiempirical approach of Schramm and Fowler did reproduce the same basic fission behavior along the $r$ process path as the detailed microscopic-macroscopic calculations of Schramm and Fiset (1973). It should be noted that all of these fission estimates were developed for neutron-rich nuclei along the valley of beta stability and out to the $r$-process path. A justification of their application out to the dripped-neutron regime would be worthy of further study.

When the temperature rises to large enough values, photodisintegration reactions $[(\gamma, \alpha)$ or $(\gamma, p)]$ will tend to rearrange the nuclei into nuclear statistical equilibrium. For simplicity, consider the photodisintegration reaction 
${ }^{A} Z+\gamma={ }^{A} Z^{\prime}+{ }^{a} q$. When the time scale $\tau_{y}$ for this reaction becomes less than $\tau_{e}$ or $\tau_{\beta}$, photodisintegration rearrangement begins to occur. Reaction theory gives (see, e.g., Clayton 1968)

$$
\tau_{y}{ }^{-1} \sim\left(\Gamma_{\text {eff }} / h\right) \exp \left[-\left(\mu_{q}+E_{0}\right) / k_{\mathrm{B}} T\right]
$$

where $\mu_{q}$ is the binding energy of $q$ in ${ }^{A} Z$ and $E_{0}=0.122\left(Z^{2} q^{2} a T_{9}^{2}\right)^{1 / 3} \mathrm{MeV}$ is the energy most favorable for nuclear reaction. $\Gamma_{\text {eff }}$ is an effective reaction width over all resonances. For the nuclei under consideration, $\Gamma_{\text {eff }} \sim 10^{-2}$ to $10^{-5} \mathrm{MeV}$. For $q=$ proton, $\mu_{q} \sim 12 \mathrm{MeV}$ and $E_{0} \sim T_{9}{ }^{2 / 3} \mathrm{MeV}$. Thus, for $T_{9}=(2,5,10)$ one finds $\tau_{y} \sim\left(10^{8}, 10^{-7}, 10^{-14}\right) \mathrm{s}$, respectively. As a result, only for $T_{9}>3$ will photodisintegration be important. However, when this occurs, a reaction network should be employed to describe the composition; the model employed here is not appropriate.

\section{RESULTS}

The equilibrium composition of the neutron star matter is determined by solving the four simultaneous equilibrium equations (8), (9), (12), and (13) for the quantities $\rho_{\text {nuc }}, n_{d}, r$, and $R$. The only input parameter is $x=Z \mid A$, which is chosen to give matter of the required density. For the shell function $S(N, Z)$ chosen, the equilibrium matter has a magic proton number, $Z=50$. Apart from shell effects, the results are identical to earlier calculations (Ravenhall, Bennett, and Pethick 1972).

The decompression process proceeds as follows. The expansion is carried out by increasing $R$ on the time scale

$$
\tau_{e}=446 \alpha \rho^{-1 / 2} \mathrm{~s}
$$

(the parameter $\alpha$ is discussed below), and satisfying the equilibrium conditions (12) and (13). The number of baryons, $B$, in the Wigner-Seitz cell is held constant and equal to $N_{d}+A$. As the matter expands and the pressure is reduced, neutrons rapidly leave the nucleus. The path followed in the $Z-N$ plane is illustrated in Figure 1 for the case of $\log (\alpha\langle\rho \mid f \tau\rangle)=-3,-4$, and -5 . The matter ceases to be in beta equilibrium $(\Delta \neq 0)$, but $\beta$-decays are disallowed, initially, because of their prohibitively long time scale, so $Z$ remains constant. Finally, $\Delta$, equation (15), becomes large enough that the $\beta$-decay rate $\tau_{\beta}{ }^{-1}\left(\propto \Delta^{6}\right)$ becomes greater than the expansion rate $\tau_{e}{ }^{-1}$. Beta decays then occur in rapid succession because of the departure of the nuclei from the magic proton number. This is exhibited in Figure 2. Beta decays are handled as follows: When $\tau_{\beta}<\tau_{e}, Z$ is increased by one, $R$ and $B$ being held constant, and (12) and (13) are used to find $\rho_{\text {nuc }}$ and $n_{d}$. This procedure is repeated until $\tau_{\beta}>\tau_{e}$, at which point the expansion is allowed to resume. Since several MeV are released per $\beta$-decay, the temperature rises quickly to a more or less constant value of $(1-2) \times 10^{9} \mathrm{~K}$, as illustrated in Figure 2 . Note that at magic proton numbers $(Z=82,114)$ the $\beta$-decay time scale rises, temporarily preventing a further change in $Z$ and allowing the temperature to adiabatically decrease. Gradually, the nuclei become more and more unstable to fission, which occurs when $\tau_{f}<\tau_{\beta}$ or $\tau_{e}$. Since rather larger energies are involved, a good approximation is to assume symmetric fission. The daughter cells have $B^{\prime}, Z^{\prime}$, and $R^{\prime}$, where $B^{\prime}=\frac{1}{2} B, Z^{\prime}=\frac{1}{2} Z$, and $R^{\prime}=R / 2^{1 / 3}$. Used with (12) and (13), the new

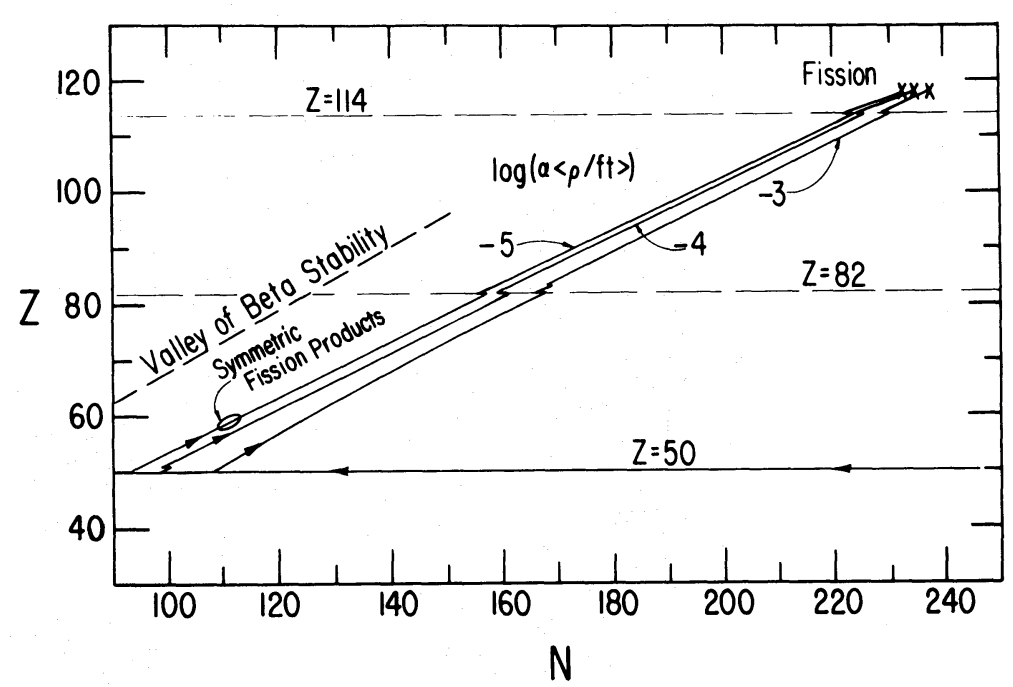

FIG. 1.-The synthesis path for nuclei in expanding, initially cold, neutron star matter. Initially $x=0.04$ and $\rho=1.8 \times 10^{14}$ $\mathrm{g} \mathrm{cm}^{-3} . Z$ and $N$ are the proton and neutron numbers of an average nucleus. Each path is labeled by its value of $\left.\log (\alpha<\rho / f \tau\rangle\right)$. The valley of beta stability and the assumed magic proton numbers 50, 82, and 114 are displayed. Fission terminates the paths at the points marked with a cross; the products of symmetric fissions are indicated by the loop. 


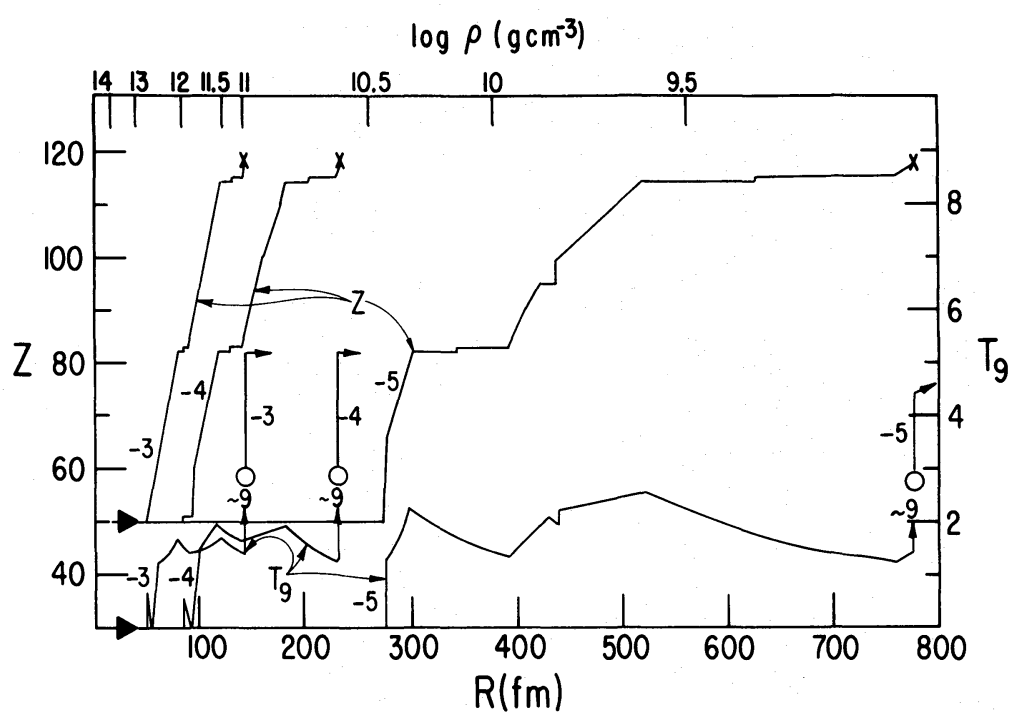

Fig. 2.-The proton number $Z$ and the temperature $T_{9}$ in units of $10^{9} \mathrm{~K}$ as a function of the Wigner-Seitz cell radius $R$ (in fm), for the calculations of Fig. 1. The corresponding mass density $\rho\left(\right.$ in $\left.\mathrm{g} \mathrm{cm}^{-3}\right)$ is also shown. Each curve is labeled by its value of log $(\alpha\langle\rho \mid f \tau\rangle)$. Fissions are indicated by a cross, fission products by a circle, and the approximate temperature reached after a fission and subsequent beta decays is displayed above the arrows.

composition of the matter may be found. The energy released in the fission, $d E_{\text {nuc }}=\frac{1}{2}\left[E_{\text {nuc }}(Z, A)-2 E_{\text {nuc }}\left(Z^{\prime}, A^{\prime}\right)\right]$, leads to a large temperature increase. From Figure 2 one sees that, after the first fission, temperatures near $10^{10} \mathrm{~K}$ are reached. As discussed in the previous section, this is large enough to initiate $(\gamma, p)$ or $(\gamma, \alpha)$ reactions. The matter will tend toward statistical equilibrium among a variety of nuclei. The model employed here, however, ceases to be valid because a reaction network is necessary to describe the composition. However, the temperature, density, and neutron-proton ratio at this point may be used in an $r$-process calculation to continue the evolutionary calculations (see $\S \mathrm{VI})$.

It is of interest to note that the lattice melting temperature

$$
T_{m}=9 Z^{2} e^{2} /(10 \gamma R) \sim(8.7 / R) \times 10^{11} \mathrm{~K},
$$

where $\gamma \sim 50$ (Mestel and Ruderman 1967) is not exceeded until after the first fission has taken place.

Some of the physical parameters of the model, such as $\tau_{e},\langle\rho / f t\rangle, \kappa, \Gamma$, and even $S$ itself, have quite uncertain values; and to ensure that the processes we have described are not affected by these uncertainties, ranges of values for them have been explored.

The effect of changing the expansion time scale relative to the $\beta$-decay time scale can be seen in Figures 1 and 2. Since $\tau_{f}$ is a very critical function of the nuclear mass, and since $\tau_{\gamma}$ depends sensitively on the temperature, this change may be carried out by varying only the parameter $\alpha$. It is clear from Figure 1 that varying $\alpha$ by even a factor of 100 does not affect the synthesis path significantly. However, the density after the first fission is quite sensitive to the quantity $\alpha\langle\rho \mid f \tau\rangle$. The significance of this is discussed in the next section.

Figure 3 displays the results of varying the quantities $\Gamma$ and $S$ for the case $\log (\alpha\langle\rho \mid f \tau\rangle)=-4$. Removing the effects of the external neutron gas on the shell function $S$ is seen to result in the appearance of neutron shell effects which were previously smeared out. Removing the effects of shells completely changes the path of the nuclei most importantly in that the initial equilibrium configuration has a different $Z$ and the lack of the magic proton number 114 affects the point at which the path is terminated due to fission.

The initial density of the matter will slightly affect the trajectory of the nuclei in the $Z-N$ plane. It has been found that the trajectory is changed only near the point where $\beta$-decays ensue. This can be explained in terms of the dependence of $\Delta$ on the density for various initial densities. As the synthesis path moves upward, this dependence on the initial density disappears. This is analogous to the behavior noted in the comparison of Figures 1 and 3 . After $\beta$-decays begin, all the paths converge to the neutron drip line.

\section{DISCUSSION}

The extremely rapid rates of neutron capture (eq. [14]) lead straightaway to the conclusion that it is virtually impossible for deuterium to form. Neutrons can be captured only after a $\beta$-decay increases $Z$, which happens on a hydrodynamic time scale. Since $\beta$-decays lead automatically to a capturing of neutrons $(x=Z / A$ remains approximately constant), and also because fissions lead to cycling, no free neutrons can escape. Even if free neutrons were 


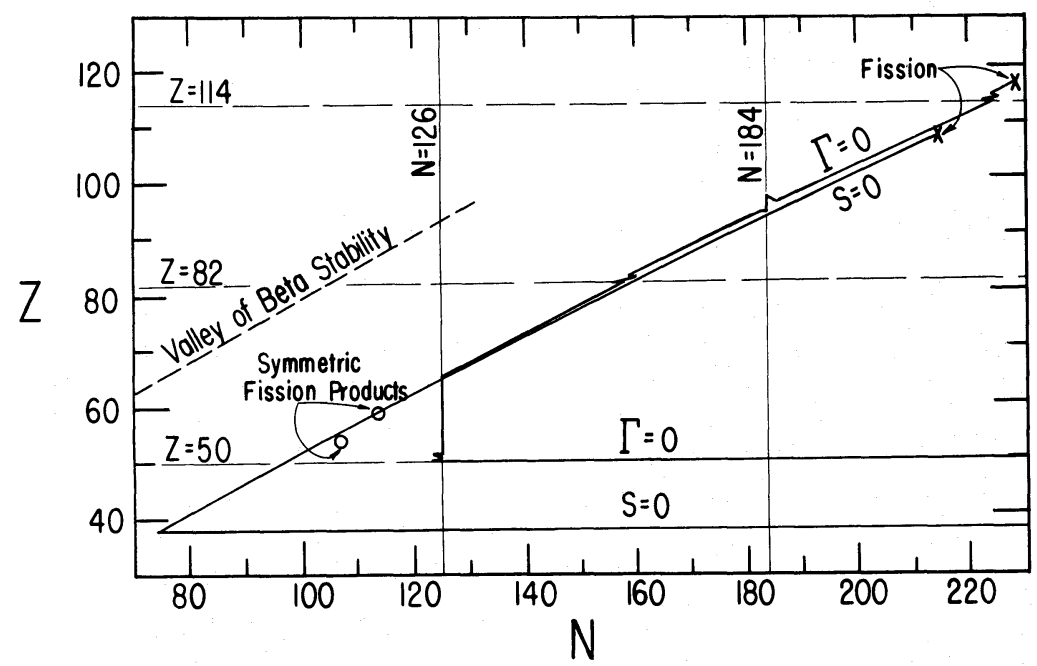

Fig. 3.- Same as Fig. 1 for the case $\log (\alpha\langle\rho \mid f \tau\rangle)=-4$. The curve $S=0$ was computed assuming all shell effects to be absent; the curve $\Gamma=0$ was calculated assuming that only the smearing of the neutron levels above the nucleus due to the external dripped neutrons was absent.

ejected, it is still possible to rule out the formation of deuterium (Epstein, Lattimer, and Schramm 1976). Since the lifetime of a neutron against capturing a proton in an essentially pure hydrogen cloud of density $\rho$ is

$$
1 / \tau \sim 4 \times 10^{4} \rho \mathrm{s}^{-1}
$$

and since free neutrons decay in about $1000 \mathrm{~s}$, the density of hydrogen required to produce much deuterium is at least $10^{-8} \mathrm{~g} \mathrm{~cm}^{-3}$. Since densities this large are normally found only in stellar envelopes, it must be concluded that deuterium is unable to be formed. Some implications of this result and others concerning deuterium are found in Epstein, Lattimer, and Schramm (1976).

An interesting feature of this mechanism is that the synthesis path lies to the right of the usual $r$-process path in the Z- $N$ plane-this is simply due to the higher density of free neutrons that exist outside the nucleus. As a result, the value of $Z$ reached before fission terminates the path will be greater than in the $r$-process with equivalent fission parameters. In Figure 1, for instance, this occurs at $Z \sim 118$ with $A \sim 350$ (which after freeze-out would decay to the $\beta$-stable nucleus with the same $A$ ).

Even if the same $Z$ were reached as in the $r$-process, the larger numbers of $\beta$-decays required to reach the valley of beta stability would result in a larger final $Z$. In view of the recent possible detection of superheavy elements (Gentry et al. 1976) with the notably large proton number $126(A \sim 350)$, the ability of this mechanism to produce larger values of $A$ is potentially important. As pointed out in the last section, the relative rapidity of the $\beta$-decay rate compared to the expansion rate may increase the density at freeze-out. The higher this density, the higher the nuclear mass produced since the synthesis path stops at approximately the same $Z$.

Our model may not be used once photodisintegrations become important. However, the temperature, density, and average neutron excess may be used as initial conditions in a dynamical $r$-process calculation, which involves a network of nuclei. Such calculations have already been carried out in the study of hot ejected neutron-rich matter (Cameron et al. 1970; Schramm 1973; Sato, Nalsayana, and Ikeuchi 1973; Sato 1974; Hillebrandt et al. 1976; Schramm and Norman 1976). The detailed composition is sensitive to $\beta$-decay time scales, explosion dynamics, and other uncertain quantities. However, from their results, it seems safe to conclude that the final composition of the ejected matter is heavy neutron-rich nuclei.

A future study will attempt to explore in more detail the required dynamic $r$-process calculations to determine how well ejected cold neutron star matter might reproduce the observed abundances of $r$-process matter. Two general trends should be noted here. First, larger neutron densities tend to shift the synthesis path to the right in the $Z-N$ plane. Second, higher temperatures tend to shift the path in the reverse direction. The nuclei finally synthesized will be determined by the two quantities $n_{d}$ and $T$ when the synthesis stops (freeze-out). Peaks in the $r$ process abundances originate from the $\beta$-decay of neutron-magic-number nuclei. The more neutron-rich the nuclei are at freeze-out, the more the $r$-process magic-number abundance peaks will be shifted to lower mass. Eventually, it may even be possible to determine the importance of black-hole-neutron-star collisions; namely, if the predicted abundances do not agree well with what is observed, the contribution of black-hole-neutron-star collisions to the enrichment of the interstellar medium may be quite small. On the other hand, if the abundances agree, this may indicate that these events ought to be studied in more detail to better determine the amount of 
ejected neutron star matter. However, at the present time, let it merely be said that neutron star ejecta can produce heavy neutron-rich nuclei and may produce somewhat heavier nuclei than a standard $r$-process.

We would like to acknowledge fruitful discussions with G. Baym, D. Lamb, C. Pethick, and R. Epstein. This research was supported in part by NSF grant AST 74-21216 and NASA grant NSG 7212 at the University of Chicago and PHY 75-21590 at the University of Illinois. One of us (J. M. L.) wishes to express his appreciation for the partial support of the Benfield Fellowship at the University of Texas at Austin.

\section{REFERENCES}

Bahcall, J. N., and Wolf, R. A. 1965, Phys. Rev., 140, B1452. Baym, G., Bethe, H. A., and Pethick, C. J. 1971, Nucl. Phys., A175, 225.

Beaudet, G., Petrosian, U., and Salpeter, E. E. 1967, Ap. J. $150,979$.

Blake, J. B., and Schramm, O. N. 1973, Ap. J., 179, 569.

Cameron, A. G. W., Truran, J. W., and Delano, M. D. 1970 Proc. International Conference on the Properties of Nuclei Far from the Region of Beta Stability, Leypin.

Clayton, D. D. 1968, Principles of Stellar Evolution and Nucleosynthesis (New York: McGraw-Hill).

Dicus, D., Kolb, E., Tubbs, D., and Schramm, D. N. 1976, Ap. J., 210, 481 .

Dyson, F. J. 1970, “Neutron Stars and Pulsars," Fermi lecture given at the Scuola Normale Superiore di Pisa (1971 Roma: Accademia Nacionale dei Lincei).

Epstein, R. I., Lattimer, J. M., and Schramm, D. N. 1976, Nature, 263, 198.

Flowers, E. 1973, Ap. J., 180, 911.

Gentry, R. V., Cahill, T. A., Fletcher, N. P., Kaufmann, H. C., Medsker, L. R., Nelson, J. W. and Flocchini, R. G. 1976, Phys. Rev. Letters, 37, 11.

Hillebrandt, W., Takahashi, K. and Kodama, T. 1976, Proc. 3d International Conference on the Properties of Nuclei Far from the Region of Beta Stability (CERN Report).
Lamb, D. Q. 1974, Ph.D. thesis, University of Rochester.

Landau, L. D., and Lifshitz, E. M. 1969, Statistical Physics, 2d ed. (Reading, Mass: Addison-Wesley).

Lattimer, J. M., and Schramm, D. N. 1976, Ap. J., $210,549$.

Mestel, L., and Ruderman, M. A. 1967, M.N.R.A.S., 136, 27.

Myers, W. D., and Swiatecki, W. J. 1966, Nucl. Phys., 81, 1.

Negele, J. W., and Vauterin, D. 1973, Nucl. Phys., A207, 298.

Ravenhall, D. G., Bennett, C. D., and Pethick, C. J. 1972, Phys. Rev. Letters, 28, 978.

Sato, K. 1974, Progr. Theor. Phys., 51, 726.

Sato, K., Nalsayana, K., and Ikeuchi, S. 1973, Progr. Theor. Phys., 49, 1166.

Schramm, D. N. 1973, Ap. J., 185, 293.

Schramm, D. N., and Fiset, E. O. 1973, Ap. J., 180, 551.

Schramm, D. N., and Fowler, W. A. 1971, Nature, 231, 103.

Schramm, D. N., and Norman, E. V. 1976, Proc. 3d International Conference on the Properties of Nuclei Far from the Region of Beta Stability (CERN Report).

Siemens, P. J., and Pandharipande, V. R. 1971, Nucl. Phys., A173, 561 .

Vautherin, D., and Brink, D. M. 1970, Phys. Letters, B32, 149.

JAMES M. LATTIMER: Department of Astronomy, University of Illinois, Urbana, IL 61801

FRED MACKIE: Department of Physics and Astronomy, Northwestern University, Evanston, IL 60201

D. G. Ravenhall: Department of Physics, University of Illinois, Urbana, IL 61801

D. N. ScḥRAmm: Enrico Fermi Institute-LASR, 933 E. 56th St., Chicago, IL 60637 